\title{
Inclusión social de actores del conflicto armado colombiano: retos para la educación superior*
}

\author{
WILLIAM PACHÓN MUÑOZ**
}

Artículo recibido: 1 de junio de 2016

Artículo aprobado: 3 de mayo de 2017

Doi: http://dx.doi.org/10.12804/revistas.urosario.edu.co/desafios/a.4917

Para citar este artículo: Pachón Muñoz, W. (2018). Inclusión social de actores del conflicto armado colombiano: retos para la educación superior. Desafíos, 30(1), 279-308. Doi: http:// dx.doi.org/10.12804/revistas.urosario.edu.co/desafios/a.4917

\section{Resumen}

El articulo plantea la discusión del proceso de reintegración a la vida social y económica de excombatientes del conflicto armado interno en Colombia y el papel de la educación superior. Para ello, desde la perspectiva de la educación inclusiva, aborda elementos conceptuales, jurídicos y políticos que han comprendido los procesos de desmovilización, desarme, reinserción y reintegración acaecidos en Colombia durante las últimas tres décadas y su relación con las instituciones de educación superior (IES).

\footnotetext{
* El presente artículo es producto de una reflexión analítica derivada del proyecto de Investigación EES-1558: "Educación Superior y post-conflicto en Colombia: proceso de reconstrucción de tejido social basado en el post-conflicto". Esta investigación fue financiada por la Universidad Militar Nueva Granada (UMNG), Bogotá, Colombia.

** Docente investigador de la Universidad Militar Nueva Granada, Facultad de Relaciones Internacionales, Estrategia y Seguridad, Bogotá, Colombia. Administrador público, especialista en Administración Financiera y candidato a magíster en Análisis de Problemas Políticos, Económicos e Internacionales Contemporáneos. Desarrolla investigaciones en: migración forzada transnacional, educación superior y posconflicto, política exterior y gobiernos subnacionales en América Latina y medios de comunicación masiva y proceso de paz en Colombia. Correo electrónico: william.pachon@unimilitar.edu.co orCID: http://orcid. org/0000-0002-4111-9441
} 
Concluye que la universidad debe contrarrestar barreras de orden político, económico y psicosocial a fin de garantizar procesos de inclusión social efectiva en un escenario de posconflicto en Colombia.

Palabras clave: Colombia, posconflicto, excombatientes, educación superior, educación inclusiva, inclusión social.

\title{
Social Inclusion of Actors in the Colombian Armed Conflict: Challenges for Higher Education
}

\begin{abstract}
The article discusses the process of reintegration of veterans of the armed conflict in Colombia into social and economic life and the role of higher education. It does so from the perspective of inclusive education and addresses conceptual and legal elements related to the process of demobilization, disarmament, and reintegration in Colombia over the past three decades and its relationship to higher education institutions. The article concludes that universities must counteract political, economic and psycho-social barriers in order to ensure effective social inclusion in a post-conflict scenario in Colombia.
\end{abstract}

Keywords: Colombia, postconflict, former combatants, bigher education, inclusive education, social inclusion.

\section{Inclusão social de atores do conflito armado colombiano: desafios para a educação superior}

\begin{abstract}
Resumo
$O$ artigo apresenta a discussão do processo de reintegração à vida social e econômica de ex-combatentes do conflito armado interna na Colómbia e a educação superior. Para isto, desde a perspectiva da educação inclusiva, aborda elementos conceptuais e juridicopolíticos que têm compreendido os processos de desmobilização, desarme, reinserção e reintegração acontecidos na Colômbia durante as últimas três décadas e a sua relação com as Instituições de Educação Superior (IES). Conclui que a Universidade deve
\end{abstract}


contra-arrestar barreiras de ordem politica, económica e psicossocial a fim de garantir processos de inclusão social efetiva em um cenário de pós-conflito na Colômbia.

Palavras-chave: Colômbia, pós-conflito, ex-combatentes, educação superior, educação inclusiva, inclusão social.

\section{Introducción}

La prolongación del conflicto armado colombiano ha oscilado históricamente entre la solución militar y la salida negociada. Esta última opción ha sido precedida o acompañada por una construcción institucional y normativa que busca posibilitar la construcción de una paz sostenible. En este sentido, y en un escenario reciente, se generaron acciones que dieron lugar a la desmovilización y reintegración a la vida civil de exintegrantes de grupos armados al margen de la ley. Estas personas recibieron beneficios jurídicos, económicos y educativos, entre otros, sin que estos les garantizaran un efectivo proceso de inclusión social.

Frente a este contexto político del país, diferentes sectores de la sociedad se cuestionan por su aporte en un eventual escenario de posconflicto y en el consiguiente proceso de construcción de paz. La universidad, como institución generadora de conocimiento, no ha sido ajena a estas problemáticas. Desde la década de 1960, al interior de las universidades, se configuraron espacios de discusión política e ideológica que convergieron con procesos de movilización estudiantil ${ }^{1}$. Algunos de estos movimientos gravitaron entre la lucha armada y la deliberación política, enmarcadas dentro de un discurso de justicia social. Por otro lado, la universidad está llamada a constituirse como un actor trascendental en la construcción de escenarios de inclusión social. Para el caso específico de las personas que pertenecieron a organizaciones al margen de la ley y se encuentran cursando un proceso de reintegración a la vida civil es importante pensar en la educación como una estrategia central de inclusión social.

\footnotetext{
1 Para un mayor acercamiento a la relación del movimiento estudiantil universitario con el conflicto y la violencia en la universidad en Colombia, puede consultarse el artículo de Acevedo-Tarazona \& Gómez (2000).
} 
En este sentido, el presente artículo propone una reflexión para todas aquellas organizaciones educativas que hacen parte de las instituciones de educación superior (IES) en Colombia y su rol como constructoras de inclusión social a través de su función formadora. Para ello, se formula la siguiente pregunta orientadora: ¿en qué medida las acciones implementadas por las IES pueden contribuir a la inclusión social de excombatientes en proceso de reintegración?

La respuesta a la anterior pregunta se estructura en seis apartados generales. El primero de ellos presenta la definición de los términos desarrollados a lo largo del artículo. Los apartados dos y tres presentan la problemática abordada y la hipótesis principal que se pretende poner en discusión. La cuarta parte describe la evolución de los procesos de desarme, desmovilización y reintegración en Colombia desde una perspectiva jurídico-política. El quinto apartado se enfoca en analizar el rol de las IES en el proceso de inclusión social de excombatientes. Finalmente, en su fase conclusiva, el artículo esboza las reflexiones sobre los retos que enfrentan las IES para garantizar la inclusión social en un escenario de posconflicto en Colombia.

\section{Elementos conceptuales para el análisis}

La noción de conflicto armado es abordada principalmente por los instrumentos de derecho internacional humanitario (DIH) que buscan reconceptualizar la palabra 'guerra'. El artículo 2, común a los Convenios de Ginebra de 1949 y el Protocolo Adicional II de esos mismos convenios, introduce este concepto y plantea la distinción entre los conflictos armados de carácter internacional y los conflictos armados de carácter no internacional.

El primer tipo de conflictos, de carácter internacional, se establece como "guerra declarada o de cualquier otro conflicto armado que surja entre dos o varias Altas Partes Contratantes, en este caso Estados, así sea que una de ellas no haya reconocido el estado de guerra" (artículo 2 común). 
Frente a los conflictos sin carácter internacional, se presentan mayores debates y complejidades. El Protocolo II, adicional a los Convenios de Ginebra de 1949, plantea que existe un conflicto armado cuando se presentan confrontaciones armadas

que se desarrollen en el territorio de una Alta Parte contratante entre sus fuerzas armadas y fuerzas armadas disidentes o grupos armados organizados que, bajo la dirección de un mando responsable, ejerzan sobre una parte de dicho territorio un control tal que les permita realizar operaciones militares sostenidas y concertadas y aplicar el presente Protocolo (Comité Internacional de la Cruz Roja —CICR—, 1977).

Complementando las nociones jurídicas, se encuentran las definiciones trabajadas por organizaciones académicas y bases de datos, como las presentadas por el profesor Uprimny en el marco del Correlates of War Project (COW) de la Universidad de Michigan, en Estados Unidos; la base de datos de la Universidad de Uppsala en Suecia, y la del PRIO, en Noruega. Según este autor, dichas bases alejándose de las definiciones jurídicas

coinciden en señalar que, para que pueda hablarse de conflicto armado en un Estado, la violencia debe (i) superar una cierta intensidad, ya que debe ocasionar al menos un cierto número de víctimas; además, debe tratarse de una violencia (ii) relativamente organizada, pues deben existir combates; lo cual supone que (iii) el actor armado que se enfrenta al Estado debe contar con una estructura militar básica, y (iv) ser capaz de ofrecer una resistencia armada que perdure al menos un cierto periodo (Uprimny, 2011).

Por su parte, la noción de posconflicto ha tomado fuerza a partir de la explosión de conflictos de carácter no internacional y de la creciente relevancia que ha tomado la resolución de los conflictos por medio de conversaciones y acuerdos de paz. A pesar de tener este contexto como referencia, para analistas del tema como Galtung (1975), Boutros-Ghali (1992), Chetail (2009) y Rettberg (2003; 2012), la noción 
de posconflicto se ha aplicado sin importar cuál sea la vía por la que se termina la confrontación. Así, el posconflicto

se define como el fortalecimiento y la solidificación de la paz para evitar una recaída en el conflicto, aunque de manera reciente, sin embargo, ha sido aplicado también a las acciones anteriores al final del conflicto que buscan atender sus causas (Ugarriza, 2013).

Este periodo de consolidación y construcción de la paz incluye otro tipo de procesos, donde el principal es el de desarme, desmovilización y reintegración (DDR) de excombatientes y los mecanismos propios de la justicia transicional, así como la solución de las causas estructurales que generaron la confrontación.

Para definir qué es un excombatiente es preciso entender la noción de combatiente. Cabe resaltar que el DIH ha construido, a partir de los convenios y sus protocolos adicionales, un Estatuto del combatiente y del prisionero de guerra, en el que es necesario el reconocimiento del primero para que pueda ser aplicado el segundo. De acuerdo con este estatuto, son combatientes "Los miembros de las fuerzas armadas de una Parte en conflicto [...], con excepción del personal sanitario y religioso y del personal militar de la protección civil" (de Preux, 1989).

Dentro de las fuerzas armadas de una parte del conflicto, asociada al Estado se encuentran: el ejército, las milicias y los cuerpos de voluntarios que formen parte del ejército o que lo constituyan: los marinos mercantes organizados para participar directamente en las hostilidades; la población de un territorio no ocupado que tome espontáneamente las armas para combatir contra las tropas invasoras, si lleva las armas a la vista y respeta las leyes y costumbres de la guerra, y las fuerzas policiales, siempre y cuando se haya notificado a las otras partes en conflicto. Estos cuerpos deben encontrarse en subordinación de una parte del conflicto y contar con una organización de tipo militar bajo un mando responsable; están obligados a respetar las normas del derecho internacional aplicables en caso de conflicto armado (de Preux, 1989). No obstante, la utilización del 
concepto de combatiente puede entenderse, en términos generales, de forma que también haga referencia a aquellas personas pertenecientes a grupos armados irregulares. En este sentido, se puede considerar como excombatiente a toda persona que haya conformado una de las partes en conflicto.

Por reintegración se asume la noción dada por la Asamblea General de la ONU como el proceso mediante el cual los excombatientes adquieren estatus civil y obtienen un empleo e ingresos sostenibles. La reintegración es esencialmente un proceso económico y social con un cronograma de tiempo abierto, que se lleva a cabo principalmente en comunidades a nivel local. Es parte del desarrollo general de un país y una responsabilidad nacional que, además, requiere de asistencia externa a largo plazo (Instituto de Estudios Geoestratégicos y Asuntos Políticos - Universidad Militar Nueva Granada, 2013).

En Colombia, la Agencia Colombiana para la Reintegración ${ }^{2}$ (ACR) es la entidad encargada de coordinar, diseñar y ejecutar con entidades públicas y privadas la política estatal dirigida a personas o grupos armados al margen de la ley, que se hayan desmovilizado voluntariamente. La misión de la ACR es impulsar el retorno de la población desmovilizada a la legalidad de forma sostenible. En este sentido, desarrolla programas de educación, formación laboral, apoyo psicosocial e impulsa nuevos proyectos productivos.

El proceso de reintegración comprende una ruta que inicia con el desarme y la desmovilización de grupos al margen de la ley y consta de tres etapas. La primera se denomina etapa básica, donde el excombatiente se reintegra a la vida civil (familiares, amigos y actores sociales); la segunda es la etapa intermedia donde se brindan condiciones económicas. Por último, se encuentra la etapa avanzada en la cual se

2 La ACR tiene su origen en el Programa de Reincorporación a la vida civil del Ministerio del Interior y de Justicia Nacional, el cual funcionó entre 2003 y 2006. En septiembre de 2006 es creada como Alta Consejería Presidencial para la Reintegración Social y Económica de Personas y Grupos Alzados en Armas y a partir del 3 de noviembre de 2011 es transformada en la Agencia Colombiana para la Reintegración. 
trabaja la sostenibilidad en la legalidad. Este proceso se conduce con un posmonitoreo y acompañamiento permanente. Asimismo, con la Ruta de Reintegración se busca brindar a las personas desmovilizadas las herramientas para que, en un futuro, cada una de ellas pueda ejercer su ciudadanía de manera autónoma y digna (Agencia Colombiana para la Reintegración, 2014).

El concepto de inclusión social puede definirse como el proceso de cambio que asegura que todas las personas, familias, comunidades e incluso regiones tengan las mismas oportunidades y posibilidades reales y efectivas de acceder, participar, relacionarse y disfrutar social, económica, política y culturalmente, junto con los demás ciudadanos, sin ninguna limitación o restricción (Congreso de la República, 2013; Araoz-Fraser, 2010).

Esta posición conceptual, que persigue un cambio, conlleva a establecer una relación entre aquello de lo cual se está excluido y lo que se quiere incluir. De ahí que investigadores sociales como Castiblanco y Gordo (2002); Brundtland, Estebañez y Martín (2002), y Huxley y Thornicroft (2003), citados en Bravo (2007), definan la inclusión a partir de su contracara, la exclusión. Esta es definida como un fenómeno multidimensional que expresa la situación de una sociedad fragmentada, dual, caracterizada por la negación o la inobservancia de los derechos sociales, económicos, políticos y culturales de un conjunto de la población, la cual se encuentra radicada en la inoperancia del Estado como garantizador de los derechos básicos de la sociedad y tiene como reflejo la fractura de la cohesión social, factor crítico para la democracia y para el desarrollo de la ciudadanía.

Para Bravo (2007), la situación de exclusión, además de ser una problemática social de acceso a bienes, servicios y procesos políticos, tiene implícito un problema de ciudadanía. En este sentido el establecimiento de acciones concretas para la inclusión social forma parte de la tendencia de ampliación progresiva de la ciudadanía (AraozFraser, 2010). En síntesis, la inclusión/exclusión social plantea tres perspectivas analíticas: 1) como una problemática social de acceso a bienes, servicios y procesos políticos; 2) como una problemática de ciudadanía por la supeditación y anteposición de los derechos de los 
individuos, y 3) como una problemática de realización de los individuos, cómo se consideran que son y están en la sociedad (Bravo, 2007).

De acuerdo con lo anterior, la noción de educación superior en relación con el proceso de inclusión social ha de entenderse como el sistema social, político, económico y cultural que atraviesa la institucionalidad educativa en función a la adaptación de los estudiantes y no viceversa. Aquí resultan pertinentes los aportes de Bermúdez, Bravo y Vargas (2009), quienes consideran que la educación superior juega un rol transformador en las sociedades en la medida en que su importancia estratégica se hace visible tan pronto como se reconocen los efectos de la incorporación de la ciencia y la tecnología, así como de la reflexión elaborada sobre sus fines en los procesos de trabajo, en la producción de la riqueza material y simbólica y en el desarrollo de la organización social. En este orden de ideas, materializar la inclusión social en términos de educación superior implica establecer una estrategia de "educación inclusiva"3 (Ministerio de Educación Nacional, 2013).

Los avances en materia de educación inclusiva a nivel internacional han sido producto de múltiples movilizaciones políticas a escala mundial, tales como la Declaración Mundial sobre Educación para Todos y el Marco de Acción para Satisfacer las Necesidades Básicas de Aprendizaje (1990), la Declaración de Salamanca (1994), la Declaración Mundial sobre la Educación Superior en el Siglo XXI (1998) y el Marco de Acción de Dakar (2000); todas manifestaciones que permitieron avanzar en el acceso a la educación superior de personas con discapacidad, a formas progresivas de inclusión de otras poblaciones en condición de vulnerabilidad ${ }^{4}$, particularmente, en aquellos

\footnotetext{
3 Para el Ministerio de Educación Nacional (2013) la educación inclusiva se define como una estrategia central para luchar contra la exclusión social y hace parte de una multiplicidad de alternativas que buscan promover e implementar el proceso aferente a la inclusión social. 4 Entiéndase por población vulnerable al grupo de personas (mujeres, niñas, campesinos, grupos étnicos, personas con discapacidad, personas en situación de desplazamiento, población víctima del conflicto armado interno y población habitante de frontera) que se encuentran en estado de desprotección o incapacidad frente a una amenaza a su condición psicológica, física, mental, social, económica, política y/o cultural, entre otras.
} 
países que presentan bajo crecimiento económico, conflicto armado e inestabilidad política (Bermudez, Bravo \& Vargas, 2009).

Estos pronunciamientos a nivel mundial legitimaron poco a poco el fundamento conceptual del Principio de Educación Inclusiva para Todos, principio que desde la Unesco se planteó como el objetivo a alcanzar por parte de todos los países del mundo, que buscó direccionar las respuestas de los Estados para suplir las necesidades diversas de los educandos y promover la participación en el aprendizaje y la reducción de la exclusión, permitiéndoles a todas las personas, sin distinción, ejercer el derecho a la educación (Unesco, 2003).

\section{Problemática}

De acuerdo con la política nacional, en Colombia se entiende la educación como un "proceso de formación permanente, personal, cultural y social que se fundamenta en una concepción integral de la persona humana, de su dignidad, de sus derechos y de sus deberes" (Congreso de la República de Colombia, 1994). Es así como, dentro de las modalidades de atención educativa a poblaciones, la Ley General de Educación (Ley 115 de 1994), en su artículo 68, reconoce la educación para la rehabilitación social. A través de esta modalidad, se desarrollan los programas ofrecidos a personas y grupos "cuyo comportamiento individual y social exige procesos educativos integrales que le permitan su reincorporación a la sociedad”.

Asimismo, la Ley General de Educación en su capítulo 5 (artículos 68 al 71) concibe la educación para la rehabilitación social como parte integrante del servicio educativo en sus distintos niveles de formación —educación formal, no formal e informal — los cuales requieren métodos didácticos, contenidos y procesos pedagógicos acordes con la situación de los educandos (Fernández, 2014).

Si bien, desde el ámbito jurídico-político colombiano se contempla el restablecimiento de la dignidad humana y la rehabilitación social de personas y grupos sociales que han estado expuestos directamente al 
conflicto armado interno, en la práctica, la relación subyacente entre el sistema de educación y el conflicto ha representado un enigma para los políticos al momento de implementar las políticas educativas.

De acuerdo con el Banco Mundial — BM- (2005), en sociedades en conflicto, las escuelas terminan siendo cómplices de este, pues es allí donde se reproducen tanto las habilidades, los valores y las actitudes, como las relaciones sociales de los grupos dominantes en la sociedad. Estudios realizados con niños y niñas de regiones con conflictos armados de diferente índole han evidenciado que la exposición a eventos violentos está relacionada con el desarrollo de agresión, intimidación escolar y otros problemas de comportamiento, así como con un mayor riesgo de involucrarse en delincuencia y violencia más tarde en sus vidas, depresión, estrés postraumático, problemas de sueño, problemas cognitivos y de somatización — como dolores de cabeza- (Chaux, 2012).

Los efectos a largo plazo que la guerra tiene en la transformación de los roles de niños y jóvenes pueden ser extremadamente difíciles de reversar sin ningún tipo de intervención educativa o terapéutica, lo que los convierte en un factor de riesgo para la "conformación de un ciclo de la violencia” y su perpetuación (World Bank, 2005; Chaux, 2012). Muestra de ello son las experiencias de El Salvador y Guatemala en Centroamérica, cuyos conflictos armados internos llegaron a su fin en los años 1992 y 1996 respectivamente, y a pesar de que en la actualidad ninguno de los dos países registra nuevas confrontaciones armadas, ambos presentan niveles extremadamente altos de violencia. La violencia en estos países no terminó, sino que se transformó (Chaux, 2012).

De esta manera, el conflicto, que ha sido conceptualizado por el BM como "desarrollo en reversa", debe ser analizado en concordancia con su impacto en el contexto del desarrollo de los Estados:

La reducción de la pobreza y la disminución de la dependencia a las exportaciones de productos primarios requieren del funcionamiento de un sistema de educación efectivo. La educación tiene un papel clave en la mediación o la profundización de los conflictos. 
A través de la educación se aumenta la capacidad de resiliencia ${ }^{5}$ de la población a enfrentar los conflictos y esta constituye una estrategia fundamental para la reconstrucción del posconflicto ${ }^{6}$ (World Bank, 2005).

En un contexto en el cual las familias y las comunidades suelen estar divididas, dispersas o debilitadas a causa de los efectos del conflicto, las instituciones educativas están llamadas a desempeñar un papel protagónico en la reconstrucción de los valores fundamentales, la instauración de nuevos principios democráticos, la recuperación de la infancia perdida y, en general, a reconstruir el tejido social y ser facilitadoras para lograr una sociedad más pacífica, cohesionada y reconciliada a fin de evitar el retorno de la violencia (World Bank, 2005).

En este orden de ideas, la solución a los conflictos representa no solo retos para la reconstrucción, sino también importantes oportunidades para la reforma de los sistemas educativos. La educación resulta ser una de las vías sociales de incorporación a la esfera civil del excombatiente, toda vez que a partir de esta se le brinda un puente para enfrentarse a las demandas laborales que la nación hace a los ciudadanos para lograr integrarse a la fuerza productiva que impulsa la economía del país (Fernández, 2014).

\section{Hipótesis}

El Estado colombiano ha adelantado durante los últimos treinta años programas tendientes a la desmovilización, desarme, reinserción y reintegración de excombatientes del conflicto armado, posibilitándoles, desde el ámbito legal, acceder a bienes y servicios entre los que se encuentra la educación superior. De esta manera, las IES han

\footnotetext{
5 Entendida como la habilidad para surgir de la adversidad, adaptarse, recuperarse y acceder a una vida significativa y productiva. El término fue adaptado a las ciencias sociales para caracterizar a aquellas personas que, a pesar de nacer y vivir en situaciones de alto riesgo, se desarrollan psicológicamente sanas y exitosas (Cabrejos Polo, 2005).

6 Traducción propia.
} 
sido llamadas a generar escenarios de inclusión social entre excombatientes, Estado y sociedad civil. Sin embargo, la materialización del proceso de reintegración en las IES ha planteado una serie de tensiones - económicas, académicas y de políticas institucionales-, las cuales emergen como barreras para garantizar la inclusión social de los excombatientes a la comunidad académica y, por ende, a la sociedad, lo que evidencia un problema de fondo relacionado con la falta de concientización, preparación y sensibilización de la comunidad académica, la sociedad y los mismos excombatientes.

\section{Contexto jurídico-político de los programas de reintegración para excombatientes en Colombia}

Desde los años noventa se iniciaron los procesos de reintegración en Colombia, el Estado ha venido ajustando los mecanismos jurídicopolíticos para facilitar las condiciones sociales, económicas y de seguridad a aquellos combatientes de grupos armados ilegales que quieren iniciar un proceso de reincorporación a la vida civil. Es así que los efectos de la Asamblea Nacional Constituyente y la promulgación de la nueva Constitución Política de Colombia en 1991 dieron un impulso importante a las políticas de paz iniciadas desde la década de los ochenta por el presidente Belisario Betancur (1982-1986) y a la consolidación de mecanismos que permitan establecer diálogos entre el Estado, los grupos al margen de la ley y la sociedad civil.

De esta manera, organismos como el Consejo Nacional de Normalización ${ }^{7}$, la Oficina Nacional de Reinserción ${ }^{8}$, la Dirección del Programa Presidencial para la Reinserción ${ }^{9}$, la Secretaría Especial para la

\footnotetext{
7 Vinculado al Departamento Administrativo de la Presidencia de la República (DAPRE), tenía como objetivos supervisar y coordinar los programas de reinserción, asignar los recursos provenientes del Fondo para la Paz a los 17 Consejos Regionales de Normalización y colaborar en las acciones de seguimiento y evaluación de los programas. Este consejo estaba constituido por tres comisiones Comité de Reinserción, Comité para la Ejecución de los Proyectos y el Comité de Seguridad (Fundación CEPEI, 2004; Presidencia de la República, 1990).

8 Establecida en el marco del Plan Nacional de Rehabilitación (PNR).

9 Adscrita al DAPRE (Presidencia de la República, 1991).
} 
Reinserción ${ }^{10}$ y la Dirección General para la Reinserción ${ }^{11}$ tuvieron su paso por este periodo, constituyendo el abanico normativo que los gobiernos de turno fueron estructurando para adelantar los procesos de paz. Los gobiernos de César Gaviria (1990-1994), Ernesto Samper (1994-1998), Andrés Pastrana (1998-2002), y luego, con modificaciones en su enfoque y aplicación, el gobierno de Álvaro Uribe (2002-2010) mantuvieron este mecanismo legal de acceso a beneficios propios de la reintegración de guerrilleros desertores.

El nuevo siglo trajo consigo una serie de dinámicas geopolíticas y económicas en el plano internacional que demandaron del Estado colombiano, a nivel interno, una mayor creatividad para encontrar caminos más efectivos, a fin de darle solución al largo conflicto que se resistió declinar al nuevo milenio. Con la Ley 782 de $2002^{12}$ se buscó establecer el diálogo y la suscripción de acuerdos con grupos armados organizados al margen de la ley tanto para su desmovilización, como para la reconciliación entre los colombianos y la convivencia pacífica (Congreso de la República de Colombia, 2002) ${ }^{13}$. Este marco jurídico fue reglamentado en 2003 con la creación del Programa para la Reincorporación a la Vida Civil (PRVC), a cargo del Ministerio del Interior y de Justicia, y que asumió funciones del Programa de Reinserción con excepción de los acuerdos de paz (Fundación CEPEI, 2004).

En este contexto, el gobierno del entonces presidente de Colombia, Álvaro Uribe Vélez, suscribe con los grupos de autodefensas el Acuerdo de Santa Fe de Ralito para adelantar una desmovilización gradual y colectiva de 37671 combatientes, la cual se extendió por

\footnotetext{
10 En 1994 esta secretaría, perteneciente a la Red de Solidaridad Social, asume las funciones del PNR.

11 En 1999 esta dirección asume la función de coordinar y dirigir la acción del Estado para el desarrollo del programa de reinserción individual y coordinar los asuntos referentes a acuerdos de paz (Presidencia de la República, 1999).

12 Retoma la Ley 418 de 1997, prorrogada y modificada por la Ley 548 de 1999 en temas relacionados con la convivencia y eficacia de la justicia.

13 Esta ley fue prorrogada y modificada sucesivamente por las leyes 1106 de 2006, 1421 de 2010 y 1738 de 2014.
} 
un periodo de cerca de tres años — 25 de noviembre de 2003-15 de agosto de 2006 - (Verdad Abierta, 2008).

En el año 2005 se promulga en Colombia la Ley 975, más conocida como Ley de Justicia y Paz, cuyo objetivo era generar el proceso de reincorporación a la vida civil de los desmovilizados. Mediante esta Ley, en su artículo 66, se reconoce la importancia de los programas de capacitación y educación al desmovilizado para acceder a proyectos o empleos productivos (Congreso de la República de Colombia, 2005).

La creación de la Alta Consejería Presidencial para la Reintegración Social y Económica de Personas y Grupos Alzados en Armas — hoy Agencia Colombiana para la Reintegración (ACR)—, en el año 2006, se propuso canalizar la política de reintegración en Colombia brindando oportunidades a las personas desmovilizadas a través de una ruta personalizada con beneficios educativos así como psicosociales, económicos y de oportunidades de generación de ingresos a fin de permitirles encontrar una vida sostenible en la civilidad (ACR, 2013).

Esta política pública de reintegración se consolida a través del documento CONPES 3554 de 2008, el cual contempla, para efectos metodológicos, dos escenarios de la reintegración: individual ${ }^{14}$ y basada en comunidades ${ }^{15}$ (DNP, 2008).

En cuanto a la educación superior, la ACR incorpora, por medio de la Resolución 008 de $2009^{16}$, lineamientos respecto a la formulación de política en educación superior para personas en procesos de reintegración, convocando así a la primera "Mesa de trabajo para propiciar

\footnotetext{
14 El escenario basado en lo individual promueve la promoción y desarrollo de capacidades y aptitudes académicas, vocacionales y ciudadanas necesarias para la interacción pacífica en la sociedad.

15 El escenario basado en las comunidades visualiza la necesidad de crear espacios de comunicación entre las comunidades receptoras y los desmovilizados a fin de crear estrategias de convivencia, construcción de ciudadanías, reconciliación y reactivación socioeconómica de las comunidades afectadas por la violencia.

16 Resolución derogada por el artículo 50 de la Resolución 163 de 2011, que fue derogada por el artículo 47 de la Resolución 754 de 2013.
} 
el acceso, permanencia y graduación de la población desmovilizada y desvinculada en la educación superior" (ODDR, 2010).

Con la Ley 1448 de 2012 se profundiza en la disposición de mecanismos que permitan consolidar la reintegración a la vida civil de los victimarios comprometidos con el proceso de paz. En este sentido, aspectos relacionados con la restitución de los bienes y las tierras a los despojados, las garantías de no repetición y la adopción de políticas, programas y proyectos para la asistencia, atención y reparación a las víctimas esbozaban la plataforma institucional para la transición de un eventual proceso de paz y el consiguiente escenario de posconflicto.

Con la suscripción del Acuerdo final para la terminación del conflicto y la construcción de una paz estable y duradera (Gobierno Nacional de la República de Colombia; FARC-EP, 2016), se establece un nuevo marco jurídico para implementar el proceso de reincorporación ${ }^{17} \mathrm{de}$ excombatientes de las FARC-EP a la vida civil en lo económico, lo social y lo político. Este proceso tiene sus propias dinámicas, actividades y cronograma, y estará liderado por el Consejo Nacional de la Reincorporación $(\mathrm{CNR})^{18}$. Un aspecto clave de este proceso de reincorporación vuelve a ser el tema de la educación con un redireccionamiento hacia los temas rurales, agrarios, de participación política y formación para el trabajo, lo cual implica una gestión descentralizada por parte de las instituciones educativas públicas y privadas a fin de consolidar la regionalización de los programas de educación en todos sus niveles con un enfoque diferencial.

\footnotetext{
17 Según el acuerdo final, la reincorporación a la vida civil será un proceso de carácter integral y sostenible, excepcional y transitorio, que considerará los intereses de la comunidad de las FARC-EP en proceso de reincorporación, de sus integrantes y sus familias, orientado al fortalecimiento del tejido social en los territorios, a la convivencia y la reconciliación entre quienes los habitan; asimismo, al despliegue y al desarrollo de la actividad productiva y de la democracia local. La reincorporación de las FARC-EP se fundamenta en el reconocimiento de la libertad individual y del libre ejercicio de los derechos individuales de cada uno de quienes son hoy integrantes de las FARC-EP en proceso de reincorporación.

18 Decreto 2027 del 7 de diciembre de 2016 por el cual se crea el Consejo Nacional de Reincorporación (Presidencia de la República de Colombia, 2016).
} 


\section{El rol de las IES en la inclusión social de los actores armados del conflicto: la educación superior en un escenario de posconflicto}

Abordar el rol de las IES con respecto a la inclusión social de excombatientes conlleva a reflexionar acerca de los desafíos que, en materia de educación superior, deberán abordarse para transitar de un escenario de postacuerdo a uno de más largo plazo y trascendencia, como lo es el posconflicto. En ese orden de ideas, el presente apartado analiza el contexto actual de las IES $^{19}$ en Colombia, haciendo énfasis en la universidad y su compromiso con la educación inclusiva, así como su incidencia en el proceso de reintegración de excombatientes a la vida civil en el marco de una educación en y para la paz.

La universidad, para Colombia, se ha venido constituyendo en el presente siglo como aquella institución que contribuye a la formación de profesionales en el marco de la pluralidad democrática, la paz sostenible y el bienestar general. Estos lineamientos se estructuran desde el Plan Decenal de Educación 2006-2016 mediante el cual se puso en práctica toda una metodología de participación colectiva que involucra a las regiones, encaminadas a la construcción prospectiva de la política de educación requerida por el país, que es liderada por el Ministerio de Educación Nacional (MEN). Es así como en materia de educación superior el plan estableció en el título II la "Educación en y para la paz, la convivencia y la ciudadanía", proponiéndose adelantar acciones que le permitan al Estado comprender de manera más profunda los procesos de desmovilización, reintegración y reconciliación a través de la educación (MEN, 2006).

En este sentido, los lineamientos de inclusión educativa definidos por el MEN se han orientado a potenciar y valorar la diversidad cultural, promover el respeto a la diferencia y garantizar la participación de la

\footnotetext{
19 De acuerdo con las estadísticas oficiales sobre las IES en Colombia, el Sistema de Aseguramiento de la Calidad de Educación Superior (SACES) indica que a diciembre de 2015 en el país existían un total de 287 IES con registro calificado, de las cuales 62 hacían parte del sector oficial, 206 no oficial y 19 de régimen especial. Del total de IES, 82 corresponden a universidades, de las que 50 pertenecen al sector privado, 31 al sector público y 1 al régimen especial (MEN - SACES, 2015).
} 
comunidad dentro de una estructura intercultural en los procesos educativos, lo que supone una transformación del sistema de educación a nivel superior en cuatro dimensiones estructurales: 1) la trascendencia de lo estrictamente académico-curricular para constituirse en lo social; 2) la conformación de una comunidad académica capaz de transformarse, entendiendo la importancia de la riqueza implícita en la diversidad estudiantil; 3) la predominancia de la defensa y protección de los derechos humanos, y 4) el inicio de una educación para todos.

Posteriormente, con la promulgación de la Ley 1732 del 1 de septiembre de 2014, la "Cátedra de Paz" establece el marco jurídico-político para que las instituciones educativas, entre ellas las IES, reglamenten esta disposición gubernamental y materialicen los objetivos trazados desde el Plan Decenal de Educación en su política interna, haciendo observancia al principio de autonomía universitaria (Congreso de la República de Colombia, 2014).

Dando cumplimiento a dicho marco jurídico-político, las IES del país han implementado progresivamente una serie de reformas institucionales para articularse a la Política Nacional de Educación con el tema de la paz. De esta forma, las IES del sector público y privado, como la Universidad de Cartagena, la Universidad de Córdoba, la Universidad de Nariño, la Universidad Nacional de Colombia, la Universidad Militar Nueva Granada, la Universidad Nacional Abierta y a Distancia (UNAD), la Universidad Pedagógica Nacional de Colombia, la Universidad de Antioquia, la Escuela Superior de Administración Pública (ESAP), la Pontificia Universidad Javeriana, la Universidad de los Andes, la Universidad del Rosario, la Universidad Santo Tomás, la Universidad Externado de Colombia, la Institución Universitaria de Envigado, la Corporación Universitaria Remington (Uniremington) y la Corporación Universitaria Empresarial de Salamanca han abierto espacios académicos para incorporar población desmovilizada, ya sea a través del otorgamiento de cupos específicos, de la reducción en los requisitos de ingreso, de la entrega de becas o de otros beneficios económicos tanto para el acceso como para el acompañamiento en procesos de permanencia (ODDR, 2013; Peña Galeano, 2015). 
Si bien estos avances en materia jurídico-política han sido liderados desde el Estado conduciendo a la transformación del sistema educativo hacia el paradigma de la educación inclusiva en la educación superior, este cambio no ha tenido la misma resonancia al interior de las IES. En la práctica, como lo señala el ODDR (2010), persiste entre estas el temor a ser estigmatizadas por recibir a estos nuevos estudiantes, así que optan por admitir a todos por igual sin establecer acciones diferenciales en los procesos de admisión ${ }^{20}$. A esto se le suman los problemas estructurales irresueltos por el país en materia de educación superior en temas como cobertura ${ }^{21}$, calidad $^{22}$ y un marcado centralismo de las IES acreditadas en alta calidad en ciudades capitales ${ }^{23}$.

Lo anterior evidencia la existencia de barreras que distorsionan los procesos de educación inclusiva y tienen implicaciones en la participación propia de todo el sistema educativo en cuanto al acceso, permanencia y egreso de grupos poblacionales que se hallan en condición de vulnerabilidad, así como también de aquella población desmovilizada ${ }^{24}$ que se encuentra en proceso de reintegración (MEN, 2013).

20 En cifras del proceso de reintegración existe una población potencial de 23031 desmovilizados con formación secundaria, que muy seguramente querrán continuar sus estudios de educación superior, sumándose a los 2763 excombatientes que para el momento se encuentran cursando su formación profesional (ACR, 2016).

21 El indicador de cobertura por parte de las IES muestra avances progresivos en cuanto el acceso a la formación superior de la población potencial que se encuentra en el rango de edad de 17 a 21 años la cual, para el 2015, ascendía a 4349823 jóvenes. Si bien el incremento porcentual de estudiantes matriculados en los niveles de formación técnica profesional, tecnológica y universitaria pasó del 31,6\% al 49,4\% correspondiente a los años 2007 y 2015 respectivamente, este avance en materia de educación superior resulta magro para afrontar el posconflicto.

22 En cuanto a calidad, tan solo el 20\% de las IES se encuentra acreditado en Alta Calidad, y de este porcentaje, el $81 \%$ lo ha obtenido las universidades — 49 en total— (CNA, 2016). 23 Se resalta la concentración del $51 \%$ de las IES acreditadas en ciudades grandes como Bogotá y Medellín mientras, con participaciones marginales, se ubican IES en las ciudades medianas y pequeñas como Cali, Cartagena, Manizales y Bucaramanga.

24 En esta categoría se clasifican a las personas menores de dieciocho años de edad, conocidas como desvinculadas, que han dejado de participar, por diferentes circunstancias, de las acciones de violencia perpetradas por algún grupo armado al margen de la ley. Y la población propiamente desmovilizada define aquellos adultos que por decisión individual abandonan 
Entre los elementos que conforman las barreras de acceso a la educación superior el MEN identificó: 1) estigmatización social de esta población por parte de la sociedad en general y de la comunidad universitaria en particular; 2) deficiencias en competencias generales y específicas que faciliten el acceso a la educación superior, y 3) insuficiencia de propuestas académicas relacionadas con un eventual escenario de posconflicto y reconciliación que promuevan el acceso de la población al sistema de educación superior. Para superar estas barreras, el MEN trazó una serie de estrategias que van desde la promoción de escenarios de diálogo entre las instituciones del Estado y las IES a fin de incentivar el acceso de esta población, hasta la conformación de grupos, semilleros, observatorios y grupos de investigación que aborden esta temática.

En cuanto a los elementos que integran las barreras de permanencia en la educación superior, el MEN identificó las siguientes: 1) dificultades de adaptación a la vida universitaria y a los espacios de sociabilidad con la comunidad académica, y 2) falta de capacitación de docentes y recursos pedagógicos adicionales. Aquí las estrategias propuestas por el MEN se orientaron a promover procesos de formación a la comunidad académica — directivos, docentes, estudiantes y personal administrativo- en temas de posconflicto y reconciliación, así como el acompañamiento académico y psicosocial al desmovilizado por parte del Estado y de las IES.

Por último, la barrera de graduación en educación superior expone la dificultad de inserción en el mercado laboral del desmovilizado; aquí, la estrategia se centraliza en el sector productivo del país y la agencia interinstitucional para financiar proyectos productivos (MEN, 2013).

Paralelo a estas estrategias propuestas por el MEN, y a fin de contrarrestar dichas barreras, la Asociación Colombiana de Universidades (ASCUN) propone acciones para las IES en un escenario de posconflicto, las cuales se resumen en las siguientes tareas: 
1) la participación activa y solidaria en el cumplimiento del pacto social; 2) el desarrollo de programas orientados a crear las condiciones de resiliencia para fortalecer la dinámica de la reconciliación; 3) el establecimiento de actividades académicas orientadas a la construcción de ciudadanía en un ambiente de confianza que fortalezca el tejido social; 4) el fomento de mecanismos de cooperación entre las instituciones sociales y las comunidades; 5) el diseño e implementación de políticas educativas de inclusión y apertura para atender a poblaciones vulnerables y a grupos afectados por el conflicto; 6) realizar diseños curriculares que se orienten a generar capacidades para la gestión del conflicto y el manejo constructivo de situaciones generadoras de violencia; $y$, 7) apoyar la dinamización y el fortalecimiento de la sociedad civil, para que en conjunto con el Estado y los sectores de la producción propicien los ambientes y suministren los recursos necesarios para el cumplimiento de los compromisos exigidos por el pacto social y participen como veedores de los mismos (ASCUN, 2014).

Asimismo, las IES deberán gestionar retos en materia de políticas, programas y proyectos sociales orientados al logro de tres objetivos: reconstrucción social, reconciliación humana y gestión de los conflictos para su resolución. En este sentido, consolidar la paz en Colombia demanda de una activa participación de todos los ciudadanos, organizaciones sociales, empresas e instituciones educativas en todos sus niveles, dándole especial énfasis a la formación de profesionales y dirigentes capaces de ponerlas en funcionamiento (ASCUN, 2014).

Esta transformación del sistema educativo a escenarios de inclusión, en concordancia con Carrillo (2013), implica el establecimiento de procesos académicos flexibles con el fin de generar acciones que no juzguen a los excombatientes, permitiéndoles retomar su proceso educativo sin temor a ser estigmatizados, posibilitándoles reconocer su pasado para resignificar en ellos la importancia del poder transformador de la educación en un contexto de paz. Asimismo, este proceso de reconstrucción psicosocial lo debe cursar la sociedad, apoyándose en la educación para visualizar a los excombatientes como actores relevantes en este proceso. 
Toda estrategia de reintegración y/o reincorporación a plantearse posterior al conflicto será pertinente siempre y cuando se fundamente en razones que comprendan la multidimensionalidad que encierra el paso a la vida civil y la diversidad misma de los excombatientes. Es así que pensar la diversidad dentro de los procesos de inclusión educativa exige partir de la premisa de proteger las particularidades de cada estudiante en el marco de una educación que realmente involucre a todos los actores de la sociedad.

Esto significa, no solamente la transformación formal de la institución educativa, sino la formación, sensibilización y concientización de toda la comunidad académica para lograr el cambio hacia ambientes educativos incluyentes en términos de paz desde un enfoque diferencial, de tal manera que a los y las excombatientes (legales-ilegales) que pertenecieron o hicieron parte directa o indirectamente de las organizaciones y/o actores del conflicto armado colombiano se les garantice un tránsito a la vida civil en cuanto a equiparación de oportunidades, como de disposición de condiciones para que puedan surtir un proceso de reparación y reconciliación consigo mismos, sus familias y la sociedad.

\section{Conclusiones}

Inclusión social de actores del conflicto armado colombiano: retos para la educación superior se presenta en un momento coyuntural para la historia política del país, contexto en el cual el gobierno de Juan Manuel Santos Calderón y las FARC-EP han logrado suscribir un acuerdo de paz después de más de cuatro años de negociación y diálogos. Es así que el cierre de este texto no puede dejar pasar inadvertido este acontecimiento, más aun cuando dentro de muchos escenarios académicos se criticaron las reflexiones que investigadores —entre los cuales me incluyo- hicimos sobre el tema del posconflicto. Así, entre las críticas recibidas, abordar el término posconflicto no era más que un neologismo con el que se pretendía idealizar un escenario hipotético de la paz sobre la tierra estéril de la guerra. 
En este contexto, y si bien el nuevo panorama resulta "regenerador" para la sociedad colombiana, pensar el posconflicto y la inclusión de excombatientes desde la educación superior configura un nivel de complejidad mayor que, sin duda, requerirá de la intervención creativa por parte de las IES públicas y privadas en Colombia a una escala multinivel: local, regional, nacional e internacional. Esto con el fin de generar estrategias, pedagogías y metodologías que contribuyan a la consolidación de la "cultura de paz" al interior de todas las estructuras y organizaciones sociales, políticas, económicas y culturales del país.

Ahora bien, y habiendo recorrido desde lo histórico, lo jurídico-político y lo conceptual los procesos de desarme, desmovilización y reintegración, así como la reapertura de los procesos de reincorporación en Colombia, surgen por lo menos tres controversias de orden político, económico y psicosocial del rol desempeñado hasta el momento por las IES en su propósito de consolidar una política nacional de educación encaminada a fortalecer la "Educación en y para la paz, la convivencia y la ciudadanía". Estas controversias a la vez se configuran como nuevas barreras a los procesos de inclusión social efectiva de excombatientes en un escenario de posconflicto en Colombia.

Desde lo político, las IES han venido adelantando arreglos institucionales que apuntan a dar cumplimento a los "compromisos" trazados desde la Política Nacional de Educación y los Lineamientos del Plan Nacional de Educación. Parte de estos arreglos se han materializado con la apertura de escenarios académicos como foros, congresos y seminarios, y la conformación de observatorios, centros de investigación, cátedra de paz, etc., así como la concertación de reuniones inter IES, las cuales han concluido en la formulación de tareas y acciones que pudiesen aportar ante un eventual acuerdo de paz y un posterior escenario de posconflicto.

En ese orden de ideas, el rol desempeñado por las IES en torno a la dinámica hacia la construcción de la paz en Colombia ha reproducido una cartografía política de la educación superior para la paz, y marca la diferencia de por lo menos dos tipos de IES en concordancia al nivel de incidencia en la definición y ejecución de la "Política de educación 
para la paz". Por un lado, se han configurado las IES élite que dominan y recrean el discurso de la paz e inciden significativamente en los fundamentos filosóficos y epistemológicos de la formulación de la "Política de educación para la paz"; mientras que, por otro lado, se encuentran las IES gregarias que a la postre serán las encargadas de implementar, ejecutar y evaluar la política en el ámbito local y regional, más que en el nacional.

Lo anterior ha permitido establecer una serie de arreglos institucionales de carácter simbiótico y a la vez isomorfo en su componente estructural, lo que ha permitido legitimar el discurso de la paz desde el centro de poder a las regiones y demás zonas periféricas. De esta manera otro aspecto a considerar en esta cartografía política de la educación superior por la paz tiene que ver con el nivel de relacionamiento e incidencia política que tiene(n) la o las IES tanto en el plano local, regional y nacional como en el internacional.

En este sentido las IES élite por lo general las conforman universidades que, dada su trayectoria y activismo político, lideran la agenda de la paz al establecer los lineamientos a seguir en esta materia, con un nivel de incidencia mayor que desciende desde el plano nacional e internacional hacia lo regional y local. Por su parte, las IES gregarias las conforman, además de universidades, las instituciones universitarias, las escuelas tecnológicas y las instituciones tecnológicas y técnicas profesionales que, al tener un reducido espacio de relacionamiento e incidencia política en los planos local, regional, nacional e internacional, resultan ser las responsables de implementar la política.

Esta relación asimétrica del rol político de las IES con los centros de gravedad del poder contrasta con el deber ser de los procesos de educación inclusiva, en la medida en que todavía se concibe la construcción de la paz desde un enfoque altamente centralizado y controlado por las IES élite para ser aplicado, sin mayor mediación, por parte de las IES gregarias en las regiones. Esto explica, en parte, la tardía participación de las IES privadas y su respectivo rezago en relación con las IES públicas, en cuanto a la incorporación de arreglos institucionales en su política interna con el fin de contribuir con acciones 
efectivas al acceso, permanencia y graduación de excombatientes y la subsiguiente construcción de la paz en Colombia.

Desde lo económico una vez más los temas de cobertura, calidad y concentración poblacional en las ciudades, así como la incipiente infraestructura física y tecnológica desarrollada a nivel regional, el abandono del campo, producto de la violencia, y la precaria institucionalidad estatal en las regiones, han incidido en que, desde criterios de sostenibilidad económica, las IES se piensen desde los ámbitos urbanos más que desde lo rural. De hecho, una de las grandes falencias en que se encuentra la educación superior en la actualidad, tiene que ver con la escaza oferta de programas académicos relacionados con temas de educación rural en todos los niveles de la formación profesional.

El reto para las IES en el escenario del postacuerdo, que ha empezado a labrar la sociedad colombiana, establece una oportunidad para resignificar la educación superior, esta vez desde las regiones. Esto exige, desde lo institucional, descentralizar sus procesos académicosadministrativos a través del rediseño organizacional que permita la concurrencia de diferentes sectores económicos, sinérgicas inter IES, inversión estatal y cooperación internacional, todo ello encaminado a la generación de nuevo conocimiento para la reactivación social, política, económica y cultural del campo. En otras palabras, una revolución agraria que derribe las barreras y asimetrías físicas y simbólicas del anacronismo estructural en que, históricamente, se ha concebido el campo respecto a la ciudad.

Finalmente, la controversia psicosocial conduce a cuestionar la discusión que hasta ahora las altas instancias y los tomadores de decisión de las IES han llevado a cabo en torno a la construcción de la paz para el país, que si bien ha sido de gran importancia en la medida en que ha permitido que sus aportes construyan imaginarios de la paz, también se hace necesario preguntarse, ¿cómo y dónde se pueden ver materializadas tales discusiones? ¿No sería aún más incluyente que los esfuerzos de las IES estuvieran dirigidos a la admisión de excombatientes en sus aulas? 
Es así como hasta el momento no se ha hecho evidente en la política institucional de las IES, y más exactamente en las que aquí se han definido como las IES élite, la adjudicación de cupos a desmovilizados y la implementación de programas con enfoque diferencial que les garanticen su permanencia y finalización de estudios. ¿Será que únicamente la inclusión que deben realizar las IES está destinada para aquellas que ofertan programas técnicos o tecnológicos, o aquellas que aún no cuentan con un amplio reconocimiento o estatus entre las IES, es decir, las IES gregarias?

¿Cómo se puede hablar de educación inclusiva, de educación para la paz y de educación en el posconflicto cuando no se piensa en los beneficios directos de tales medidas para los excombatientes? Su participación no puede limitarse a ser receptores pasivos de beneficios ofrecidos por las instituciones.

Por consiguiente, si se piensa en una educación inclusiva con enfoque diferencial y que permita validar procesos de reconstrucción del tejido social, la reforma al sistema de educación se hace imperativa, pues converge como elemento fundamental en donde las estrategias para reducir el riesgo de conflicto o su profundización, deberán cimentarse en el desarrollo de competencias ciudadanas - emocionales, cognitivas y comunicativas - que permitan abordar y entender la condición humana a partir de la otredad como parte constitutiva de la existencia de lo social. En este sentido, las IES representan ese nodo articulador de sentido en todo proceso de formación del ser humano, el cual le permite hacer y sentirse parte de algo, como actor transformador de su realidad.

El tema de la educación superior va a tener un manejo más complejo, dado que ya no se habla de un escenario hipotético sino de uno real, en el cual se deben pensar pedagogías creativas a fin de consolidar los puntos del acuerdo en las regiones y territorios, y generar procesos más dinámicos tendientes a paliar esas deficiencias que preexisten a nivel regional como el tema del analfabetismo o el tema del abandono del campo. Estos son retos y desafíos que, en materia de educación superior, se deben incorporar, pues no solo se trata de ver cómo se reciben en las ciudades, sino 
de pensar cómo se mantienen en los territorios que antes eran de guerra. ¿Cómo se reincorporan a los espacios que eran antes de la guerra? Las instituciones públicas y privadas deben contemplar cambios en su política institucional para permitir el fortalecimiento e implementación de los acuerdos de paz a nivel nacional y local.

\section{Referencias}

Acevedo-Tarazona, A., \& Gómez, F. J. (2000). Conflicto y violencia en la universidad en Colombia. El proyecto modernizador y el movimiento estudiantil universitario en Santander, 1953-1980. Reflexión Política, 2(4), 1-11.

Agencia Colombiana para la Reintegración. (2016). La reintegración en Colombia "Construimos paz desde el territorio". Agencia Colombiana para la Reintegración (ACR). Bogotá: Agencia Colombiana para la Reintegración.

Agencia Colombiana para la Reintegración. (2014). Evolución del Proceso de Reintegración. Fortaleza Institucional basada en la experiencia y lecciones aprendidas. Bogotá.

Araoz-Fraser, S. (2010). Contexto conceptual. En S. Araoz-Fraser, \& E. F. Central (Eds.). Inclusión social: un propósito nacional para Colombia. (pp. 1-50) Bogotá: Departamento de Economía.

Asociación Colombiana de Universidades Ascun. (1 de abril de 2014). Educación superior y posconflicto. Aportes para la construcción de una política. Boletín No. 4.

Bravo Delgado, M. F. (2007). Dimensiones de los procesos de inclusión/ exclusión social de personas con enfermedad mental. (Tesis de maestría, Bogotá, D.C., Colombia).

Bermúdez, G. I., Bravo, M. F., \& Vargas, D. R. (2009). Discapacidad en Colombia: un reto en la educación superior inclusiva. Revista Colombiana de Rehabilitación, 8(1)1-49.

Cabrejos Polo, J. (2005). La promoción de la resilencia y el diseño de políticas sociales. Facultad de Ciencias Económicas de la Universidad Nacional Mayor de San Marcos, 47-70.

Carrillo, J. F. (2013). Informe parcial de investigación acerca de medidas tomadas por distintos países para la incorporación de excombatientes a la vida civilpor medio 
de iniciativas educativas especificas y normas expedidas para lograrlo. Bogotá: MEN República de Colombia.

Chaux, E. (2012). Contribución a la educación a la construcción de paz: retos y avances. En A. Rettberg, \& U. d. Andes (Eds.) Construcción de paz en Colombia (pp. 493-512) Bogotá: Uniandes.

CNA. (2016). Histórico acreditaciones institucionales. Bogotá: Consejo Nacional de Acreditacion CNA.

Colombia, Congreso de la República. (8 de febrero de 1994). Ley 115. Ley general de educación. Título III Modalidades de atención educativa a poblaciones.

Colombia, Congreso de la República. (23 de diciembre de 2002). Ley 782. Por medio de la cual se prorroga la vigencia de la Ley 418 de 1997, prorrogada y modificada por la Ley 548 de 1999 y se modifican algunas de sus disposiciones.

Colombia, Congreso de la República. (25 de julio de 2005). Ley 975. Por la cual se dictan disposiciones para la reincorporación de miembros de grupos armados organizados al margen de la ley, que contribuyan de manera efectiva a la consecución de la paz nacional y se dictan otras disposiciones para acuerdos humanitarios. Diario Oficial.

Colombia, Congreso de la República. (27 de febrero de 2013). Ley Estatutaria 1618 de 2013. Recuperado de http:/ / wsp.presidencia.gov.co/ Normativa/Leyes/Documents/2013/LEY\%201618\%20DEL\%20 27\%20DE\%20FEBRERO\%20DE\%202013.pdf

Colombia, Congreso de la República de Colombia. (1 de septiembre de 2014). Ley 1732 Por la cual se establece la Cátedra de paz en todas las instituciones educativas del país.

Colombia, Gobierno Nacional de la República, FARC-EP. (24 de noviembre de 2016). Mesa de conversacionespara la terminación del conflicto y la construcción de una paz estable y duradera en Colombia. Recuperado de https://www. mesadeconversaciones.com.co/

Colombia, Ministerio de Educación Nacional, MEN. (2006). Lineamientos para la educación superior. Gerencia Plan Nacional de Educación. Plan Nacional Decenal de Educación 2006-2016.

Colombia, MEN. (2013). Lineamientos Política de Educación Superior Inclusiva.

Colombia, Presidencia de la República. (1 de febrero de 1990). Decreto 314. Por el cual se crea el Consejo Nacional de Normalización. 
Colombia, Presidencia de la República. (26 de diciembre de 1991). Decreto 2884. Dirección del Programa Presidencial para la Reinserción.

Colombia, Presidencia de la República. (23 de diciembre de 1999). Decreto 2646. Dirección General para la Reinserción.

Colombia, Presidencia de la República. (7 de diciembre de 2016). Decreto 2027 del 7 de diciembre de 2016 Por el cual se crea el Consejo Nacional de Reincorporación.

Comité Internacional de la Cruz Roja, CICR. (8 de junio de 1977). Protocolo II. Conflictos no-internacionales. Ginebra, Suiza.

Fernández, P. (2014). Antecedentes históricos del M-19 y la educación en la inclusión social. Recuperado de https://www.academia.edu/9964446/ FARC_Proceso_de_Paz_Guerrilla_M-19_y_Proceso_de_Paz_Educaci $\%$ C3\%B3n_y_conflicto_armado_M-19_Pol\%C3\%ADtica_ Educaci\%C3\%B3n_M-19_Desmovilizaci\%C3\%B3n_M-19_Educaci\%C3\%B3n_M-19_Desmovilizados_M-19_Pedagog\%C3\%ADa_ Inclusi $\%$ C3\% 33 n_S

Fundación CEPEI. (2004). Procesos de reincorporación en Colombia. Recuperado de https://www.verdadabierta.com/documentos/historia/ guerrillas/855-reincorporacionencolombia

Instituto de Estudios Geoestratégicos y Asuntos Políticos-Universidad Militar Nueva Granada. (2013). Desarme, desmovilización y reintegración, DDR: Una introducción para Colombia. Cuaderno de Análisis (01/13).

MEN, SACES. (2015). Estadísticas de educación superior. Bogotá: Ministerio de Educación Nacional - SACES.

Observatorio de Procesos de Desarme, Desmovilización y Reintegración, ODDR. (2010). Educación superior y reintegración. Recuperado de http:// www.observatorioddr.unal.edu.co/productos_academicos/nuevos/ educacion_superiory_reintegracionI-2010.pdf

Observatorio de Procesos de Desarme, Desmovilización y Reintegración, ODDR. (2013). La educación superiory los procesos de reintegración en Colombia. Recuperado de http:/ /www.humanas.unal.edu.co/observapazyconflicto/files/6714/3075/1947/ODDR_OIM_Educacion_Superior_y_Reintegracion_Genera_DF.pdf

Peña Galeano, G. (2015). El rol de las Instituciones de Educación Superior (IES) frente al posconflicto 2014 - 2015. Bogotá: Universidad Militar Nueva Granada. 
de Preux, J. (1989). Estatuto de combatiente y de prisionero de guerra. Revista Internacional de la Cruz Roja.

Ugarriza, J. E. (2013). La dimensión política del posconflicto: discusiones conceptuales y avances empíricos. Colombia Internacional, 77, 141-176.

Unesco. (2003). Superar la exclusión mediante planteamientos integrales en la educación. Un Desafío \& una Visión. París, Francia.

Uprimny, R. (2011). ¿Existe o no conflicto armado en Colombia? Recuperado de http://otramiradadelconflicto.wikispaces.com/file/view/ $\mathrm{EXISTE}+\mathrm{O}+\mathrm{NO}+\mathrm{CONFLICTO}+\mathrm{ARMADO}+\mathrm{EN}+\mathrm{COLOMB}$ $\mathrm{OA}+-+$ Rodrigo+Uprimny.pdf

Verdad Abierta. (2008). La desmovilización: el proceso de paz (2003-2006). Recuperado de http://www.verdadabierta.com/la-historia-de-lasauc/244-la-historia/auc/54-periodo4

World Bank. (2005). Reshaping the future. Education and postconflict reconstruction. Washington, D.C. 